\title{
$\begin{array}{ll}\text { Research Square } & \text { Preprints are preliminary reports that have not undergone peer review. } \\ \text { They should not be considered conclusive, used to inform clinical practice, }\end{array}$ or referenced by the media as validated information.
}

\section{Is there a Trade-off between Economy and Task Goal Variability in Transfemoral Amputee Gait?}

I-Chieh Lee ( $\square$ ilee5@ncsu.edu )

UNC/NCSU Joint Department of Biomedical Engineering https://orcid.org/0000-0001-7094-6270

\section{Bretta Fylstra}

UNC/NCSU Joint Department of Biomedical Engineering

Ming Liu

UNC/NCSU BME: UNC/NCSU Joint Department of Biomedical Engineering

Tommaso Lenzi

The University of Utah

He Huang

UNC/NCSU BME: UNC/NCSU Joint Department of Biomedical Engineering

\section{Research}

Keywords: mechanical energy, goal equivalent manifold, transfemoral amputee, energy recovery rate, walking

Posted Date: July 13th, 2021

DOl: https://doi.org/10.21203/rs.3.rs-659520/v1

License: (c) (i) This work is licensed under a Creative Commons Attribution 4.0 International License. Read Full License

Version of Record: A version of this preprint was published at Journal of NeuroEngineering and Rehabilitation on March 18th, 2022. See the published version at https://doi.org/10.1186/s12984-02201004-8. 
2 Is there a trade-off between economy and task goal variability in transfemoral amputee gait?

I-Chieh Lee ${ }^{a *}$, Bretta L. Fylstra ${ }^{a}$, Ming Liua ${ }^{a}$ Tommaso Lenzi ${ }^{b}$, He (Helen) Huanga

a Joint Department of Biomedical Engineering, North Carolina State University and

6 University of North Carolina at Chapel Hill, Raleigh, NC, USA

${ }^{b}$ Department of Mechanical Engineering and Robotics Center, The University of Utah, Salt Lake City, Utah, USA

*Corresponding Author: I-Chieh Lee-Engineering Building III, NC State University, 


\section{Abstract}

23 Background: Energy cost minimization has been widely accepted to regulate gait. Optimization

24 principles have been frequently used to explain how individuals adapt their gait pattern.

25 However, there have been rare attempts to account for the role of variability in this optimization

26 process. Motor redundancy can enable individuals to perform tasks reliably while achieving

27 energy optimization. However, we do not know how the goal-irrelevant and goal-relevant

28 variability is regulated.. In this study, we investigated how unilateral transfemoral amputees

29 regulate step and stride variability based on the task to achieve energy economy.

Methods: Nine individuals with unilateral transfemoral amputation walked on a treadmill at

31 speeds of $0.6,0.8,1.0,1.2$ and $1.4 \mathrm{~m} / \mathrm{s}$ using their prescribed passive prostheses. We calculated the

32 step-to-step and stride-to-stride variability and applied goal equivalent manifold (GEM) based

33 control to decompose goal-relevant and goal-irrelevant manifold. To quantify the energy

34 economy, the energy recovery rate $(\mathrm{R})$ was calculated based on potential energy and kinetic

35 energy. Comparisons were made between GEM variabilities and commonly used standard

36 deviation measurements. A linear regression model was used to investigate the trade-off between

$37 \quad \mathrm{R}$ and GEM variabilities.

38 Results: Our analysis shows greater variability along the goal-relevant manifold compared to the 39 goal-irrelevant manifold $(\mathrm{p}<.001)$. Moreover, our analysis shows lower energy recovery rate for 40 amputee gait compared to nonamputee gait (at least $20 \%$ less at faster walking speed). We found

41 a negative relationship between energy recovery rate and goal-irrelevant variability. Compared to

42 the standard deviation measurements, the variability decomposed using GEM reflected the 43 preferred walking speed and the limitation of the passive prosthetic device. 
44 Conclusion: Individuals with amputation cleverly leverage task redundancy, regulating step and 45 stride variability to the goal equivalent manifold (GEM). This result suggests that task

46 redundancy enables unilateral amputees to benefit from motor variability in terms of energy

47 economy. The differences observed between prosthetic step and intact step support the 48 development of prosthetic leg capable of enhancing positive work during the double support

49 phase and of powered prosthesis controllers that allow for variability along the task space while

50 minimizing variability that interferes with the task goal. This study provides a different

51 perspective on amputee gait analysis and challenge the field to think differently about the role of 52 variability.

53

54

55 Keywords: mechanical energy, goal equivalent manifold, transfemoral amputee, energy

56 recovery rate, walking

57

58

59

60

61

62 

computational models $(3,4)$ suggest that individuals select their preferred step length $(4,5)$, step width(6), step frequency(3), and walking speed $(1,2)$ to minimize the energy cost of walking.

67 Energy optimization characterizes both the normal and the pathological gait, acting as an important

68 factor in clinical applications $(7,8)$. Individuals with gait impairments operate close to their 69 maximum level of effort and are prone to fatigue even at low walking speeds $(9,10)$. Hence, 70 reducing the energy cost of walking is an important goal for rehabilitation $(8,11,12)$ and a key 71 metric in assessing the effectiveness of assistive devices such as prosthesis, orthosis, and 72 exoskeletons $(13,14)$

74 gait pattern. However, there have been rare attempts to account for the role of variability in this 75 optimization process. Variability is ubiquitous in motor performance $(15,16)$. Yet, a deviation 76 from the average gait pattern might cause a divergence from energy optimality $(17,18)$. The fact 77 that variation is inevitable raises a question that Is human variability regulated to assist the energy 78 optimization process? If yes, then how?

Traditionally, movement variability has been linked to noise or error, and a large variability 80 is considered "bad motor control". This concept might be influenced by traditional statistical and 81 assessment methods of movement variability (e.g., standard deviation) that assume randomness 82 and independence of observations $(16,19)$. Opposite to this traditional perspective, variability can 83 be seen to give rise to equifinality, allowing infinite number of ways to perform the same task(20, 84 21). Thus, individuals can tolerate movement variability that does not interfere with the task goal 
85 without requiring extra effort $(22,23)$. These observations suggest that variability and movement 86 redundancy can enable individuals to perform complex tasks reliably and repeatedly, increasing

87 adaptability in motor performance $(20,24)$.

Researchers have analyzed movement redundancy and variability with different

89 approaches, including uncontrolled manifold (UCM) analysis(24, 25), minimum intervention 90 principle (MIP)(23, 26), and goal equivalent manifold (GEM)(21, 27). Despite methodological

91 differences, all these approaches decompose the total variability at the body-level into goal92 relevant variability and goal-irrelevant variability. The goal-relevant variability is the variability 93 component tangent to the manifold, which does not affect the task goal. The goal-irrelevant 94 variability is the variability component perpendicular to the manifold, which causes deviation from 95 the task goal. Experiments focusing on quiet standing(24, 28), walking(29, 30), reaching(21, 23), 96 and aiming(27) show that individuals preferentially constrains the goal-irrelevant variability rather 97 than the goal-relevant variability, most likely because the latter is not detrimental to the 98 achievement of the task goal. If we assume that goal-irrelevant variability interferes with the task 99 goal and that this interference requires extra effort to correct, then higher goal-irrelevant variability 100 should bel inked to increased energy cost. Based on this rationale, there may be a trade-off between 101 energy optimization and regulation of task variability.

103 goal-irrelevant and goal-relevant variability emerge to reconcile with minimal energy cost. This 104 problem is especially interesting in individuals with unilateral lower-limb amputations, who only 105 have indirect control of the motion of the prostheses. Lower-limb amputees spend more metabolic 106 energy than nonamputee individuals during walking and more proximal amputations are associated 107 with greater metabolic energy cost than distal amputations $(12,31)$. The increased energy cost of 
108 walking might be related to a lack of ankle push-off power from the prosthesis. Individuals with 109 amputations compensate for the lack of push off power by pulling the thigh forward at the end of 110 stance. This compensatory movement allows for continuous forward propulsion but is highly 111 inefficient $(14,31,32)$. In addition, walking with a passive prosthesis requires greater mechanical 112 work for the step-to-step transition from prosthetic to intact limb, increasing the overall energy 113 cost of walking. Individuals with unilateral amputation need to adapt at every step to respond to external 115 (i.e., lack of push-off) and internal (i.e., compensatory hip movements) perturbations. Thus, 116 knowing how individuals control the step/ stride variability would be beneficial to understand how 117 they achieve optimal energy cost. The purpose of this study is to investigate how individuals with 118 unilateral transfemoral amputation walking with a passive prosthesis regulate step and stride 119 variability. Specifically, we aim to understand how goal-relevant and goal-irrelevant variability 120 are regulated with respect to energy economy. In this study, the amputee participants were asked 121 to walk on a treadmill at different speeds using their prescribed passive prostheses. We applied 122 GEM-based control to decompose the goal-relevant and goal-irrelevant variability(30, 33). Using 123 this method, we found a goal-equivalent manifold containing all the possible stride/step time and 124 stride/step length combinations that equally satisfy the goal function (details see Figure 1 and 125 methods). To quantify the energy economy, we calculated the energy recovery rate for each step 126 based on potential energy $\left(E_{p}\right)$ and kinetic energy $\left(E_{k}\right)(34)$. To optimize the recovery of 127 mechanical energy, the $E_{p}$ and $E_{k}$ curves must have the same shape, be equal in amplitude, and be 128 opposite in phase, as in a pendulum. A higher energy recovery rate indicates more efficient walking 129 (see Figure 2B and methods for details). 
We hypothesized that if individuals with amputations regulate their step/stride variability

131

132

133

134

135

136

137

138

139

140

141

142 differently about the role of variability.

\section{Methods}

\section{Experimental protocol and data collection}

explicitly to minimize energy cost, then goal-relevant variability would be larger than goalirrelevant variability given that these movement variations do not interfere with task performance. Moreover, we hypothesized that if individuals with amputations regulate their step/stride variability explicitly to minimize energy cost and the variability that interferes with task performance requires extra effort to correct, then there will be a trade-off between goal-irrelevant variability and the energy recovery rate across different walking speeds. To the best of our knowledge, this is the first study to analyze the gait variability at the level of task goal in connection to energy optimization in individuals with lower-limb amputations. Given that human behavior is goal driven, instead of focusing on common gait features such as step mean and standard deviation, we took the task goal into consideration. We believe that the results of this study will provide a different perspective on amputee gait analysis and challenge the field to think

The data collection protocol has been carefully documented in Hood et al. (2020) (35), and data has been shared on Springer Nature, scientific data. The present study examined unilateral amputees walking on the treadmill who comfortably walked at or above $0.8 \mathrm{~m} / \mathrm{s}$ were classified as full community ambulators (K3) (35). Seven male and two female transfemoral amputees (39.56 \pm 12.5 years old) participated after providing institutional-approved informed consent. The time since amputation was at least five years for all participants and participants self-reported using 
151 the prosthesis for at least 5 hours a day. The details of the demographic data are provided in Table 1521.

Each participant was asked to walk on a treadmill with their daily passive prostheses at 154 speeds of $0.6,0.8,1.0,1.2$ and $1.4 \mathrm{~m} / \mathrm{s}$. During acceleration and deceleration of the treadmill, 155 participants were instructed to hold on to the treadmill handrails. After the treadmill reached the 156 constant speed, the participants were encouraged to walk without holding the handrails and then 157 recording started. For each treadmill speed, five trials contained around 10 continuous strides 158 were recorded except for TF01 having four trials at 0.6, 0.8 and $1.0 \mathrm{~m} / \mathrm{s}$ and TF02 having 4 trials 159 at speed $0.6 \mathrm{~m} / \mathrm{s}$. Only TF5 and TF6 held the handrails all the times during the $1.4 \mathrm{~m} / \mathrm{s}$ 160 condition. During walking, a 10-camera motion capture system (Vicon, Oxford, UK) was used to 161 record the reflective markers position at $200 \mathrm{~Hz}$ and a split-belt Bertec fully instrumented 162 treadmill (Bertec Co; Columbus, $\mathrm{OH}$ ) was used to record the bilateral ground reaction forces at $1631000 \mathrm{~Hz}$. Reflective markers were placed on the participants following a modified Plug-in-Gait $164 \operatorname{Model}(36,37)$. Marker trajectories and ground reaction force data were synchronized, recorded, 165 and pre-processed using Vicon Nexus 2 software. A low-pass Butterworth filter with a cut-off 166 frequency of $6 \mathrm{~Hz}$ was applied for the marker trajectories. Inverse dynamic of rotational, 167 translational and potential energy of 15 segments (head, thorax, pelvis, and left and right 168 forearms, upper arms, hands, thighs, shanks, feet) were calculated using Visual 3D. 


\section{Data analysis}

173

174

175

176

177

178

179

180

181

182

183

184

185

186

187 188 formulated as:

$189-\frac{L_{T M}}{2}<\sum_{n=1}^{N} L_{n}-v T_{n}<+\frac{L_{T M}}{2}$

where $\mathrm{n}$ is each step/stride, $\mathrm{L}_{\mathrm{n}}$ is step/stride length, $\mathrm{T}_{\mathrm{n}}$ is step/stride time, the summation of $L_{n}-$

$191 v T_{n}$ is the overall displacement walked over $\mathrm{N}$ steps and $\mathrm{L}_{\mathrm{TM}}$ is the treadmill length. The simplest 192

Stride-to-stride and step-to-step analysis were both performed in this study. For each stride, $\mathrm{n}$, stride time $\left(\mathrm{T}_{\mathrm{n}}\right)$ was taken as the time between consecutive right heel strikes. Stride length $\left(\mathrm{L}_{\mathrm{n}}\right)$ was taken as the anterior-posterior distance travelled during each stride using the position of right and left heel markers. Stride speed $\left(S_{n}\right)$ was calculated as $S_{n}=L_{n} / T_{n}$. Step analysis was calculated using the same methods and taken the time and length between heel contact to the contralateral side heel contact. Prosthetic step was determined from prosthetic heel contact to intact heel contact where the intact limb provided push-off power during the initial double support phase and then transferred to the prosthetic single support phase. Intact step was determined from intact heel contact to the prosthetic heel contact where the prosthesis leg provided push-off power during the initial double support phase and then transferred to the intact leg single support phase. (see Figure 2B).

\section{Goal Equivalent Manifold (GEM)}

We applied a GEM based method to decompose the goal-relevant and goal-irrelevant variabilities on treadmill walking as originally proposed by Dingwell (33). The primary goal for treadmill walking with speed $v$ is to not walk off the treadmill. Thus, treadmill walking can be

(Equation 1) 
$194 \quad L_{n}-v T_{n}=0 \rightarrow \frac{L_{n}}{T_{n}}=v$

(Equation 2)

195 Hence, all $\left[L_{n}, T_{n}\right]$ pairs that satisfied Equation 2 defines the GEM, which was a red solid line in 196 the $L_{n}$ versus $T_{n}$ plane (see Figure 1A).

197 To analyze walking dynamics relative to the GEM, we first normalized each $L_{n}$ and $T_{n}$ to unit 198 variance $(\sigma=1)$ to provide an intuitive reference for comparison by dividing its own standard 199 deviation:

$200 \quad \widetilde{T_{n}}=T_{n} / \sigma\left(T_{n}\right)$ and $\widetilde{L_{n}}=L_{n} / \sigma\left(L_{n}\right)$

201 This yielded a GEM defined by the dimensionless walking speed: $\tilde{v}=$ mean $\left(\widetilde{L_{n}} / \widetilde{T_{n}}\right)$. We then 202 define the new coordinates centered at a mean "preferred operating point":[ $\left.\widetilde{T_{n}} * \widetilde{L_{n}} *\right]=$ $203\left[\operatorname{mean}\left(\widetilde{T_{n}}\right), \widetilde{v} \widetilde{T_{n}} *\right]$ and re-expressed as $\widetilde{T_{n}}{ }^{\prime}=\widetilde{T_{n}}-\widetilde{T_{n}} *$ and $\widetilde{L_{n}}=\widetilde{L_{n}}-\widetilde{L_{n}} *$

204 Finally, the goal-equivalent deviation along the GEM ( $\delta_{\mathrm{T}}$ is the goal-relevant deviation), and non205 goal-equivalent deviations perpendicular to the GEM ( $\delta_{\mathrm{p}}$ is the goal-irrelevant deviation) were 206 calculated as:

$207 \quad\left[\begin{array}{l}\delta_{T} \\ \delta_{p}\end{array}\right]=\frac{1}{\sqrt{1+v^{2}}}\left[\begin{array}{cc}1 & v \\ -v & 1\end{array}\right]\left[\begin{array}{c}\widetilde{T}_{n}^{\prime} \\ \widetilde{L_{n}}\end{array}\right]$ (Equation4)

208 To quantify how variability was distributed relative to the GEM, the standard deviation of each 209 new time series $\left(\delta_{\mathrm{T}}\right.$ and $\left.\delta_{\mathrm{p}}\right)$ was calculated (see Figure 1B). 


\section{Energy recovery rate}

216 can be measured from the gravitational and the kinetic energy of each segment calculated at each

217 instant of time $(t)$ relative to the frame of reference (Figure 2):

218 The whole-body potential energy was calculated as:

$219 \quad \mathrm{E}_{p}(\mathrm{t})=\sum_{i=1}^{S}\left(m_{i} g h_{i}(t)\right)$

(Equation 5)

220 where $h_{i}$ and $m_{i}$, respectively, are the vertical distance of center of mass to the ground and the mass

221 of the $\mathrm{i}^{\text {th }}$ segment, relative to the frame of global reference; $g$ is the gravitational acceleration (9.81

$222 \mathrm{~m} / \mathrm{s})$.

223 The whole-body kinetic energy was calculated as:

224

$E_{k}(t)=\sum_{i=1}^{n}\left(\frac{1}{2} m_{i}\left[v_{x i}^{2}(t)+v_{y i}^{2}(t)+v_{z i}^{2}(t)\right]+\frac{1}{2} m_{i}\left[k_{x i}^{2} \omega_{x i}^{2}(t)+k_{y i}^{2} \omega_{y i}^{2}(t)+k_{z i}^{2} \omega_{z i}^{2}(t)\right]\right)$ 
226 where $v_{i}$, is the linear velocity relative to the frame of the global references in $\mathrm{x}, \mathrm{y}$ and $\mathrm{z}$ axis; $k_{i}$

227 and $\omega_{i}$, respectively, are the moment of inertia about the axis through the center of mass and

228 angular velocity of the $\mathrm{i}^{\text {th }}$ segment (frame of local reference).

229 Thus, the total energy can be computed as:

$230 \quad \mathrm{E}_{\text {total }}(\mathrm{t})=\mathrm{E}_{p}(\mathrm{t})+\mathrm{E}_{k}(\mathrm{t})$

(Equation 7)

231 Over the stride and step period, the energy exchange was computed according to

232 Winter(34). The energy recovery rate $(\mathrm{R})$ represents the percentage of mechanical energy recovery

233 via exchange between kinetic and potential energy in the COM movement. This is computed as:

$234 R=100 \cdot \frac{\left(w_{n e t}-w_{\text {ext }}\right)}{w_{\text {net }}}$

235

(Equation 8)

236 where $w_{\text {ext }}=\sum_{i=1}^{N}\left|\Delta E_{p}+\Delta E_{k}\right|$ during $N$ sample period , and $\quad w_{n e t}=\sum_{i=1}^{N}\left(\left|\Delta E_{p}\right|+\right.$

$\left.237\left|\Delta E_{k}\right|\right)$ if one assumes no energy exchange between potential and kinetic energy to calculate the 238 work done by a segment during $\mathrm{N}$ sample of period.

239 In an ideal energy recovery mechanism, the work associated with changes of potential energy is

240 exactly the same as the work associated with kinetic energy changes, but with different sign:

$241 \mathrm{~W}_{\mathrm{p}}=-\mathrm{W}_{\mathrm{k}}$. That means that work produced to increase the potential energy can be obtained by

242 reducing the kinetic energy and can again be returned to increase the kinetic energy at the next

243 step-to-step transition. Hence, the larger value of R indicates better energy economy. 


\section{$248 \quad$ Statistics}

We first performed one-way repeated ANOVA to exam the commonly used gait features

250 across walking speeds (mean and standard deviation (SD) of stride/ step length and time). The

251 trend of the effect on speed was tested using within-subject contrast on a linear, quadratic, cubic,

252 and order 4. To answer the question of the first hypothesis, the differences between the two types

253 of decomposed variabilities ( $\delta \mathrm{T}$ and $\delta \mathrm{p}$ ) across five walking speeds were tested using 2-way

254 repeated ANOVA (2 variabilities X 5 speeds) in stride, intact step, and prosthesis step. To answer

255 the second question, we performed a simple linear regression of energy recovery rate (R) as the

256 dependent variable and $\delta$ p and walking speed as the independent variable for each stride, prosthetic

257 step, and intact step (model: $R_{i}=\beta 0+\beta 1 \delta p_{i}+\beta 2$ speed $\left._{i}+\varepsilon_{i}\right)$. If the coefficient of $\delta p$ is negative

258 and reaches a significant level, it indicates a trade-off between $\mathrm{R}$ and $\delta \mathrm{p}$. To understand the role of

259 push-off for prosthetic and intact leg in respect to the energy exchange, a follow-up analysis was

260 also conducted. We performed 2-way repeated ANOVA (2 (intact \& prosthesis step) * 5 (Speeds))

261 to compare the energy recovery rate between step types and speeds. The significant level was set

262 at $\alpha=.05$. For the main effects that reached the significance level, the Bonferroni test was used for 263 the post hoc comparisons. 


\section{Results}

\section{Primary gait features across walking speeds}

Figures $3 \mathrm{~A}$ and $3 \mathrm{~B}$ depict the standard deviations (SD)of the length and time of prosthetic step, intact step, and stride, respectively. For the length SD of prosthetic step, intact step, and stride

269 linearly decreased with faster walking speed (Figure 3C) (prosthetic step: $F(4,32)=11.629$, p<.001, 270 $\eta_{\mathrm{p}}^{2}=.592$, linear effect $\mathrm{F}(1,8)=24.876, \mathrm{p}<.001, \eta_{\mathrm{p}}^{2}=.757$; intact step: $\mathrm{F}(4,32)=6.826, \mathrm{p}<.001$,

$271 \eta_{\mathrm{p}}{ }^{2}=.460$, linear effect $\mathrm{F}(1,8)=22.238, \mathrm{p}<.001, \eta_{\mathrm{p}}{ }^{2}=.735$; stride: $\mathrm{F}(4,32)=6.646, \mathrm{p}<.001, \eta_{\mathrm{p}}{ }^{2}$ $272=.454$, linear effect $\left.\mathrm{F}(1,8)=14.889, \mathrm{p}<.01, \eta_{\mathrm{p}}^{2}=.650\right)$. The time variability of prosthetic step, 273 intact step, and stride also linearly decreased with faster walking speed (Figure3D) (prosthetic step: $274 \mathrm{~F}(4,32)=51.593, \mathrm{p}<.001, \eta_{\mathrm{p}}^{2}=.866$, linear effect $\mathrm{F}(1,8)=158.259, \mathrm{p}<.001, \eta_{\mathrm{p}}{ }^{2}=.9527$; intact 275 step: $\mathrm{F}(4,32)=29.234, \mathrm{p}<.001, \eta_{\mathrm{p}}^{2}=.785$, linear effect $\mathrm{F}(1,8)=51.545, \mathrm{p}<.001, \eta_{\mathrm{p}}{ }^{2}=.866 ;$ stride: $\mathrm{F}(4,32)=53.982, \mathrm{p}<.001, \eta_{\mathrm{p}}^{2}=.871$, linear effect $\left.\mathrm{F}(1,8)=117.232, \mathrm{p}<.01, \eta_{\mathrm{p}}^{2}=.936\right)$.

277

\section{Gait Variability Decomposed Based on GEM} task relevant $\left(\delta_{\mathrm{T}}\right)$ variability was significantly larger than task irrelevant $\left(\delta_{\mathrm{P}}\right)$ variability in stride, 
285 prosthetic step, and intact step (stride: $\mathrm{F}(1,8)=47.885, \mathrm{p}<.001, \eta_{\mathrm{p}}{ }^{2}=.857$; prosthetic step: $\mathrm{F}(1,8)$ $286=158.198, \mathrm{p}<.001, \eta_{\mathrm{p}}{ }^{2}=.952$; intact step: $\left.\mathrm{F}(1,8)=172.667, \mathrm{p}<.001, \eta_{\mathrm{p}}{ }^{2}=.956\right)$. For the interaction 287 effect between speed and variability, there was a significant difference in prosthetic step $(\mathrm{F}(4,32)$ $\left.288=6.827, \mathrm{p}<.001, \eta_{\mathrm{p}}{ }^{2}=.46\right)$ and a marginal level on stride $\left(\mathrm{F}(4,32)=2.670, \mathrm{p}=.051, \eta_{\mathrm{p}}{ }^{2}=.26\right)$. The 289 post hoc analyses for $\delta_{\mathrm{T}}$ in prosthetic step showed that at the speed of $1.0 \mathrm{~m} / \mathrm{s}, 1.2 \mathrm{~m} / \mathrm{s}$ and $1.4 \mathrm{~m} / \mathrm{s}$ 290 were equal but were significantly higher than the speed at 0.6 and $0.8 \mathrm{~m} / \mathrm{s}$. $\delta_{\mathrm{p}}$ in prosthetic step, on 291 the other hand, showed a trend as an U shape that walking speed at $1.0 \mathrm{~m} / \mathrm{s}$ was significantly 292 smaller than $0.6 \mathrm{~m} / \mathrm{s}$ and $0.8 \mathrm{~m} / \mathrm{s}$ and significantly smaller than $1.2 \mathrm{~m} / \mathrm{s}$ and $1.4 \mathrm{~m} / \mathrm{s}$. The post hoc 293 analyses for $\delta_{\mathrm{T}}$ in stride showed that $1.2 \mathrm{~m} / \mathrm{s}$ was significantly smaller than $1.0 \mathrm{~m} / \mathrm{s} \mathrm{and} 1.4 \mathrm{~m} / \mathrm{s}$, 294 and $\delta_{\mathrm{p}}$ showed $1.2 \mathrm{~m} / \mathrm{s}$ was significantly larger than $1.0 \mathrm{~m} / \mathrm{s}$ and $1.4 \mathrm{~m} / \mathrm{s}$.

\section{Energy recovery rate}

Figures 5 depicts the energy recovery rate of prosthetic step and intact step. There was a 300 significant interaction between step types and speed $\left(\mathrm{F}(4,32)=5.552, \mathrm{p}=.002, \eta_{\mathrm{p}}{ }^{2}=.40\right)$, and the 301 post hoc analysis indicated only in prosthetic step at speeds $1.2 \mathrm{~m} / \mathrm{s}$ and $1.4 \mathrm{~m} / \mathrm{s}$ were significantly 302 larger than other speeds. The second hypothesis was whether there is a trade-off between $\delta p$ and energy recovery rate (negative relationship). We performed a linear regression between $\delta p$, speed,

304 and energy recovery rate of prosthetic step and intact step. The results showed the coefficient in 
305 prosthetic step and intact step reached significant differences with a negative coefficient (See Table $3062)$.

307

308

Figure 5 Inserts Around Here

309

310

311

312

Table 2 Inserts Around Here

313

314

315

316

317

318

319

320 


\section{Discussion}

322

323

324

325

326

327

328

The purpose of this study was to investigate how individuals with unilateral transfemoral amputation regulate step and stride variability on the goal-relevant and goal-irrelevant manifold to achieve energy economy. We examined the hypothesis that if amputees are regulating variability in terms of task goal, a large portion of goal-relevant variability would be observed. In addition, we examined whether there would be a trade-off between goal-irrelevant variability and energy recovery rate across different walking speeds under the assumption that individuals require more energy to correct for the goal-irrelevant variability.

Looking at the main results of primary gait features, the standard deviation of stride/step length and time linearly decreased with faster walking speeds (Fig. 3A and 3B). This trend was consistent for both prosthesis steps and intact steps. While the standard deviation quantified the magnitude of deviation from the mean of all strides/steps, the time series of goal-relevant $\left(\delta_{\mathrm{T}}\right)$ and goal-irrelevant $\left(\delta_{\mathrm{p}}\right)$ variabilities exhibited the step-to -step change in respect to the task goal with the temporal order. $\delta_{\mathrm{T}}$, qualitatively, showed larger amplitudes than the $\delta_{\mathrm{p}}$ (Figure $1 \mathrm{~B}$ ) and the variabilities of $\delta_{\mathrm{T}}$ were significantly higher than the $\delta_{\mathrm{p}}$ across all speeds (Figure 4). These results support our first hypothesis and indicate that amputees explicitly regulate step/stride variability tuned to the goal equivalent manifold (GEM). Our findings on unilateral amputees followed the same trend as healthy young adults $(33,38)$ and elderly $(39,40)$. The ability to leverage the task redundancy could be critical when tasks become more demanding. For example, walking on uneven terrain requires individuals to adjust each step rather change their average gait pattern. In addition, this concept can be used as a guiding principle for designing fault-tolerant controller for powered prostheses. To achieve this goal, a powered prosthesis controller should maximize the 
343 variability along with the $\delta_{\mathrm{T}}$ while minimizing the $\delta_{\mathrm{p}}$. Moreover, the powered prosthesis controller

344 should only correct errors distributed on the $\delta_{\mathrm{p}}$ because these errors interfere with task performance.

345 In contrast, errors that vary along with the $\delta_{\mathrm{T}}$ should be tolerated as they do not interfere with the

346 task performance A prosthesis controller following this principle may improve safety while

347 reducing control effort.

The goal-relevant $\left(\delta_{\mathrm{T}}\right)$ and goal-irrelevant $\left(\delta_{\mathrm{p}}\right)$ variabilities demonstrated a different speed effect compared to the standard deviation of stride/step length and time, and such effects reflect

350 the unique characteristics of amputee gait (Figure 4). During the prosthetic step, the intact leg 351 regulates the push-off power, which propels the body forward, and the swing movement. Thus, the 352 intact leg largely determines the step time and step length. Our results show that the variability of $353 \delta_{\mathrm{T}}$ and $\delta_{\mathrm{p}}$ changed with walking speed forming an inverted $\mathrm{U}$ and $\mathrm{U}$ shape, respectively (Figure 354 4B). A similar trend was observed with healthy adults $(33,38)$. This trend suggests that the intact 355 leg can adjust the $\delta_{\mathrm{T}}$ and $\delta_{\mathrm{p}}$ variabilities that tuned to the preferred walking speed for unilateral 356 amputees (around $1 \mathrm{~m} / \mathrm{s}$ ) (41). Interestingly, the intact step did not show the same adaptability to 357 walking speed. During the intact step, the passive prosthetic leg provides limited push-off power $358(32,42)$ and swing time adaptability. Thus, although microprocessor-controlled knee prostheses 359 can adjust the joint damping to walking speed $(43,44)$, our results indicate that this adaptation 360 mechanism is not enough to achieve similar task performance to the intact leg. Future work could 361 test whether the U-shaped relationship observed in the intact leg occur in powered prostheses 362 capable of imitating the intact-leg push-off powered and swing time adaptability.

In this study, we quantified energy recovery rate at each step based on potential energy $\left(E_{p}\right)$ 364 and kinetic energy $\left(E_{k}\right)(34)$. Previous studies has assessed the effectiveness of the pendulum 
mechanism during human walking by measuring the fraction of the total mechanical energy that is recovered as a result of the transduction between $E_{p}$ and $E_{k}(45,46)$. To optimize the recovery

367 of mechanical energy, which leads to the most efficiency walking pattern, the $E_{p}$ and $E_{k}$ curves 368 must have the same shape, be equal in amplitude, and be opposite in phase, as in a pendulum. A 369 higher energy recovery rate indicates more efficient walking (see Figure $2 \mathrm{~B}$ and methods for 370 details). Previous studies showed that for healthy adults at preferred walking speed $(\sim 1.6 \mathrm{~m} / \mathrm{s})$, as 371 much as $70 \%$ of the required external mechanical energy can be recovered due to this energy 372 saving mechanism $(45,47,48)$. The other $30 \%$ of external mechanical energy is lost from the 373 system and must be supplied by the muscles(49). Our study shows that unilateral amputees have 374 lower recovery rate than healthy adults. At $1.4 \mathrm{~m} / \mathrm{s}$, the average energy recovery rate during the 375 prosthetic step was about $40 \%$. Moreover, the recovery rate could be up to $60 \%$ depending on the 376 amputee individual (see Figure 5). This result explains why individuals with amputation using a 377 passive prosthesis spend, on average, more energy during walking than nonamputee individuals.

As expected, the prosthetic step had a larger energy recovery rate than the intact step.

379 Interestingly, the speed trends for the prosthetic and intact steps were different. The prosthetic step 380 showed a linearly increase of the energy recovery rate with walking speed, whereas the intact step 381 showed an inverted U-shaped profile (Figure 5). This difference in the speed trends could be due 382 to the different ability of the intact and prosthetic leg to generate positive work during push-off. 383 Previous studies in healthy adults found an inverted U-shaped relationship, although for a wider 384 range of walking speeds than the one used in this study (i.e., $0.5-2.5 \mathrm{~m} / \mathrm{s} \mathrm{vs.} 0.8-1.4 \mathrm{~m} / \mathrm{s})(45,47$, 385 48). In these studies, energy recovery rate gradually increased from $0.6 \mathrm{~m} / \mathrm{s}$ to $1.6 \mathrm{~m} / \mathrm{s}$, which is 386 similar to the walking speed range of our study This result is in agreement with our finding in the 387 prosthetic side. 

energy changes are larger than the kinetic energy changes. When walking speed increases, the kinetic energy changes increase relative to the potential energy changes, exceeding the potential energy changes after the walking speed is above the preferred walking speed. Thus, the energy recovery rate reaches its maximum for the self-selected speed and it is lower than its maximum both above and below the self-selected speed (45). Our study shows that when the intact leg is in charge of forward propulsion (i.e., prosthesis step), the amplitude of kinetic energy continuously increases along with walking speed. However, when the prosthetic leg is in charge of forward propulsion (i.e., intact-leg step), the capacity of increasing the amplitude of kinetic energy relative to the potential energy reaches a limit after the walking speed is above the amputee's preferred 398 walking speed. We speculate that this inability to increase the amplitude of kinetic energy might 399 be due to the passive prosthesis generating less power than the intact leg during push-off, resulting 400 in net negative work during the intact $\operatorname{step}(32,42,50)$. The increase of energy dissipation during 401 double support phase at fast speed might explains the inverted U-shaped relationship in a smaller 402 range of walking speed. We speculate that, with proper control of propulsive torque, powered 403 prostheses $(14,42)$ could enable amputees to achieve better energy recovery rate. Future studies 404 should investigate the step-to-step change of energy recovery rate with powered prostheses to test 405 this hypothesis.

$407 \mathrm{R}$ calculated extracted from each stride and step. Using this regression, we found that adding the $408 \delta_{\mathrm{p}}$ in the linear model significantly explains the energy recovery rate. Moreover, the negative 409 coefficient of the linear regression supports that there is the trade-off relationship between $\delta_{\mathrm{p}}$ 410 variability and recovery rate. Since the fitting considered the temporal order with $\delta_{\mathrm{p}}$ and the energy 
411 recovery rate, this result demonstrates that individuals with unilateral amputations leverage

412 movement redundancy to correct small deviations at each step to control the task variability while

413 minimizing energy cost. This short-term control mechanism (step-to-step) exploits the inherent

414 task redundancy enabling unilateral amputees to receive potential benefits from motor variability

415 in achieving energy economy. This result could inform the future design and control of powered

416 lower-limb prostheses. For example, a powered prosthesis controller that enables variations along

417 the goal-equivalent manifold may improve energy efficiency in amputee locomotion. This idea

418 could be the object of future work for rehabilitation engineers.

419 We recognize that the energy recovery rate might not accurately estimate the energy cost 420 of walking, especially for fast walking speeds (32). Although measuring the oxygen consumption 421 can lead to a more accurate estimate of the energy cost of walking, it cannot provide step-to-step

422 cost, which is needed for the proposed analysis of GEM variabilities. Using more elaborate 423 musculoskeletal models might provide a better estimation of the energy cost of walking; however, 424 inverse dynamics calculations have been shown not to solve this problem easily(51) and the current 425 validity of more elaborate musculoskeletal models does not warrant success with such an approach 426 either. We believe the interaction of task variability and energy recovery rate could provide some 427 clarification on the issue. Further interpretation would be highly speculative and require more 428 studies.

\section{Conclusion}

This study investigated how individuals with unilateral transfemoral amputation walking

431 with a passive prosthesis regulate step and stride variability on the goal-relevant and goal432 irrelevant manifolds. Our results suggest that individuals with amputation cleverly leverage task 
433 redundancy, regulating step and stride variability to the goal equivalent manifold (GEM). This 434 result suggests that task redundancy enables unilateral amputees to benefit from motor variability 435 in terms of energy economy. The differences observed between prosthetic step and intact step 436 support the development of prosthetic leg capable of enhancing positive work during the double 437 support phase. Moreover, the results of this study motivate the development of powered prosthesis 438 controllers that allow for variability along the task space while minimizing variability that 439 interferes with the task goal. This study provides a different perspective on amputee gait analysis 440 and challenge the field to think differently about the role of variability.

441

442

443

444

445

446

447

448

449

450

451

452 
454 1. Browning RC, Baker EA, Herron JA, Kram R. Effects of obesity and sex on the energetic 455 cost and preferred speed of walking. Journal of Applied Physiology. 2006;100(2):390-8.

$4562 . \quad$ Ralston HJ. Energy-speed relation and optimal speed during level walking. Internationale 457 Zeitschrift für angewandte Physiologie einschließlich Arbeitsphysiologie. 1958;17(4):277-83.

458 3. Zarrugh M, Todd F, Ralston H. Optimization of energy expenditure during level walking. 459 European journal of applied physiology and occupational physiology. 1974;33(4):293-306.

460 4. Minetti AE, Alexander RM. A Theory of Metabolic Costs for Bipedal Gaits. Journal of 461 Theoretical Biology. 1997;186(4):467-76.

462 5. Sekiya N, Nagasaki H, Ito H, Furuna T. Optimal walking in terms of variability in step 463 length. Journal of Orthopaedic \& Sports Physical Therapy. 1997;26(5):266-72.

464 6. Donelan JM, Kram R, Kuo AD. Mechanical and metabolic determinants of the preferred 465 step width in human walking. Proceedings Biological sciences. 2001;268(1480):1985-92.

466 7. Gordon NF, Gulanick M, Costa F, Fletcher G, Franklin BA, Roth EJ, et al. Physical 467 activity and exercise recommendations for stroke survivors: an American Heart Association 468 scientific statement from the Council on Clinical Cardiology, Subcommittee on Exercise, 469 Cardiac Rehabilitation, and Prevention; the Council on Cardiovascular Nursing; the Council on 470 Nutrition, Physical Activity, and Metabolism; and the Stroke Council. Circulation.

$471 \quad 2004 ; 109(16): 2031-41$.

472 8. Waters RL, Mulroy S. The energy expenditure of normal and pathologic gait. Gait 473 Posture. 1999;9(3):207-31.

474 9. Stoquart G, Detrembleur C, Lejeune TM. The reasons why stroke patients expend so 475 much energy to walk slowly. Gait \& Posture. 2012;36(3):409-13. 
476 10. Genin JJ, Bastien GJ, Franck B, Detrembleur C, Willems PA. Effect of speed on the 477 energy cost of walking in unilateral traumatic lower limb amputees. European Journal of Applied 478 Physiology. 2008;103(6):655.

479 11. da Cunha IT, Lim PA, Qureshy H, Henson H, Monga T, Protas EJ. Gait outcomes after 480 acute stroke rehabilitation with supported treadmill ambulation training: A randomized 481 controlled pilot study. Archives of Physical Medicine and Rehabilitation. 2002;83(9):1258-65.

482 12. Waters R, Perry J, Antonelli D, Hislop H. Energy cost of walking of amputees: the 483 influence of level of amputation. J Bone Joint Surg Am. 1976;58(1):42-6.

484 13. Zhang J, Fiers P, Witte KA, Jackson RW, Poggensee KL, Atkeson CG, et al. Human-in485 the-loop optimization of exoskeleton assistance during walking. Science. 2017;356(6344):12804864.

487 14. Herr HM, Grabowski AM. Bionic ankle-foot prosthesis normalizes walking gait for 488 persons with leg amputation. Proceedings of the Royal Society B: Biological Sciences. $489 \quad 2012 ; 279(1728): 457-64$.

490 15. Bernstein NA. Dexterity and its development: Psychology Press; 2014.

491 16. Newell KM, Corcos DM. Variability and motor control: Human Kinetics Publishers 492 Champaign; 1993.

493 17. Wong JD, Selinger JC, Donelan JM. Is natural variability in gait sufficient to initiate 494 spontaneous energy optimization in human walking? Journal of neurophysiology. $495 \quad 2019 ; 121(5): 1848-55$.

496 18. Malatesta D, Simar D, Dauvilliers Y, Candau R, Borrani F, Préfaut C, et al. Energy cost 497 of walking and gait instability in healthy 65-and 80-yr-olds. Journal of applied physiology. $498 \quad 2003 ; 95(6): 2248-56$. 
499 19. Stergiou N, Decker LM. Human movement variability, nonlinear dynamics, and

500 pathology: Is there a connection? Human Movement Science. 2011;30(5):869-88.

501 20. Latash ML, Scholz JP, Schöner G. Motor Control Strategies Revealed in the Structure of 502 Motor Variability. Exercise and Sport Sciences Reviews. 2002;30(1):26-31.

503 21. Cusumano JP, Dingwell JB. Movement variability near goal equivalent manifolds:

504 Fluctuations, control, and model-based analysis. Human movement science. 2013;32(5):899-923.

505 22. Todorov E. Optimality principles in sensorimotor control. Nat Neurosci. 2004;7(9):907-

50615.

507 23. Todorov E, Jordan MI. Optimal feedback control as a theory of motor coordination.

508 Nature neuroscience. 2002;5(11):1226-35.

509 24. Hsu W-L, Scholz JP, Schöner G, Jeka JJ, Kiemel T. Control and Estimation of Posture

510 During Quiet Stance Depends on Multijoint Coordination. Journal of Neurophysiology.

$511 \quad 2007 ; 97(4): 3024-35$.

512 25. Freitas SM, Scholz JP, Latash ML. Analyses of joint variance related to voluntary whole-

513 body movements performed in standing. Journal of neuroscience methods. 2010;188(1):89-96.

514 26. Liu D, Todorov E. Evidence for the flexible sensorimotor strategies predicted by optimal 515 feedback control. Journal of Neuroscience. 2007;27(35):9354-68.

516 27. Cusumano JP, Cesari P. Body-goal variability mapping in an aiming task. Biological 517 cybernetics. 2006;94(5):367-79.

518 28. Hsu W-L, Scholz JP. Motor abundance supports multitasking while standing. Human 519 movement science. 2012;31(4):844-62. 
520 29. Black DP, Smith BA, Wu J, Ulrich BD. Uncontrolled manifold analysis of segmental

521 angle variability during walking: preadolescents with and without Down syndrome.

522 Experimental brain research. 2007;183(4):511-21.

523 30. Dingwell J, Cusumano JP, Cavanagh P, Sternad D. Local dynamic stability versus

524 kinematic variability of continuous overground and treadmill walking. J Biomech Eng.

$525 \quad 2001 ; 123(1): 27-32$.

526 31. Quesada RE, Caputo JM, Collins SH. Increasing ankle push-off work with a powered

527 prosthesis does not necessarily reduce metabolic rate for transtibial amputees. J Biomech.

$528 \quad 2016 ; 49(14): 3452-9$.

529 32. Houdijk H, Pollmann E, Groenewold M, Wiggerts H, Polomski W. The energy cost for

530 the step-to-step transition in amputee walking. Gait \& posture. 2009;30(1):35-40.

531 33. Dingwell JB, John J, Cusumano JP. Do humans optimally exploit redundancy to control 532 step variability in walking? PLoS Comput Biol. 2010;6(7):e1000856.

533 34. Winter DA. Biomechanics and motor control of human movement: John Wiley \& Sons; 5342009.

535 35. Hood S, Ishmael MK, Gunnell A, Foreman KB, Lenzi T. A kinematic and kinetic dataset 536 of 18 above-knee amputees walking at various speeds. Scientific Data. 2020;7(1):150.

537 36. Limited VMS. Plug-in Gait Reference Guide. Vicon Documentation. 2016.

538 37. Phinyomark A, Petri G, Ibáñez-Marcelo E, Osis ST, Ferber R. Analysis of Big Data in

539 Gait Biomechanics: Current Trends and Future Directions. Journal of medical and biological 540 engineering. 2018;38(2):244-60. 
541 38. Roerdink M, de Jonge CP, Smid LM, Daffertshofer A. Tightening Up the Control of

542 Treadmill Walking: Effects of Maneuverability Range and Acoustic Pacing on Stride-to-Stride

543 Fluctuations. Frontiers in Physiology. 2019;10(257).

544 39. Decker LM, Cignetti F, Potter JF, Studenski SA, Stergiou N. Use of Motor Abundance in 545 Young and Older Adults during Dual-Task Treadmill Walking. PLOS ONE. 2012;7(7):e41306.

546 40. Dingwell JB, Salinas MM, Cusumano JP. Increased gait variability may not imply 547 impaired stride-to-stride control of walking in healthy older adults: Winner: 2013 Gait and

548 Clinical Movement Analysis Society Best Paper Award. Gait \& Posture. 2017;55:131-7.

549 41. Batten HR, McPhail SM, Mandrusiak AM, Varghese PN, Kuys SS. Gait speed as an 550 indicator of prosthetic walking potential following lower limb amputation. Prosthet Orthot Int. $551 \quad 2019 ; 43(2): 196-203$.

552 42. Fylstra BL, Lee IC, Huang S, Brandt A, Lewek MD, Huang H. Human-prosthesis 553 coordination: A preliminary study exploring coordination with a powered ankle-foot prosthesis. 554 Clinical Biomechanics. 2020;80:105171.

555 43. Michael J. Prosthetic suspensions and components. Atlas of amputations and limb 556 deficiencies: Surgical, prosthetic, and rehabilitation principles 3rd ed Rosemont (IL): American 557 Academy of Orthopaedic Surgeons. 2004:409-25.

558 44. Gard SA. The influence of prosthetic knee joints on gait. Handbook of Human Motion: 559 Springer International Publishing; 2018. p. 1359-82.

560 45. Cavagna G, Willems P, Heglund N. The role of gravity in human walking: pendular 561 energy exchange, external work and optimal speed. The Journal of Physiology. 2000;528(3):65756268. 
563 46. Cavagna GA, Thys H, Zamboni A. The sources of external work in level walking and 564 running. The Journal of physiology. 1976;262(3):639-57.

565 47. Willems PA, Cavagna GA, Heglund NC. External, internal and total work in human 566 locomotion. The Journal of experimental biology. 1995;198(Pt 2):379-93.

567 48. Cavagna G, Willems PA, Legramandi M, Heglund NC. Pendular energy transduction 568 within the step in human walking. Journal of Experimental Biology. 2002;205(21):3413-22.

569 49. Cavagna GA, Heglund NC, Taylor CR. Mechanical work in terrestrial locomotion: two 570 basic mechanisms for minimizing energy expenditure. American Journal of Physiology-

571 Regulatory, Integrative and Comparative Physiology. 1977;233(5):R243-R61.

572 50. Kuo AD. Energetics of actively powered locomotion using the simplest walking model.

573 Journal of biomechanical engineering. 2002;124(1):113-20.

574 51. Gitter A, Czerniecki J, Weaver K. A reassessment of center-of-mass dynamics as a 575 determinate of the metabolic inefficiency of above-knee amputee ambulation. American journal 576 of physical medicine \& rehabilitation. 1995;74(5):332-8. 
584 Ethics approval and consent to participate

585 The experimental protocol for this study was approved by the University of Utah Institutional

586 Review Board.

\section{Consent for publication}

588 All subjects provided written informed consent, including written permission to publish photos

589 and videos of the experiment.

590 Availability of data and materials

591 The data has published at scientific data

592 Acknowledgements and Funding

593 This work was partly supported by the National Institutes of Health [NIH EB024570,

594 R01HD098154] and by the Department of the Army [W81XWH-16-1-0701].

595 Author information

596 Affiliations

597 Joint Department of Biomedical Engineering, North Carolina State University and

598 University of North Carolina at Chapel Hill, Raleigh, NC, USA

599 I-Chieh, Bretta L. Fylstra, Ming Liu, He Huang

600

601

602

Department of Mechanical Engineering and Robotics Center, The University of Utah, Salt

603 Tommaso Lenzi

604

605

606 Correspondence to $\underline{\text { I-Chieh Lee }}$

Corresponding Author

607 Contributions

608 IL and BLF processed the data. TL directed the data collection. ML and IL discussed the results. 609 IL drafted the manuscript. HH, ML,BLF. and TL revised the manuscript. All authors read and 610 approved the final manuscript prior to publication.

611 Competing interests

612 The authors declare no competing interests. 
A

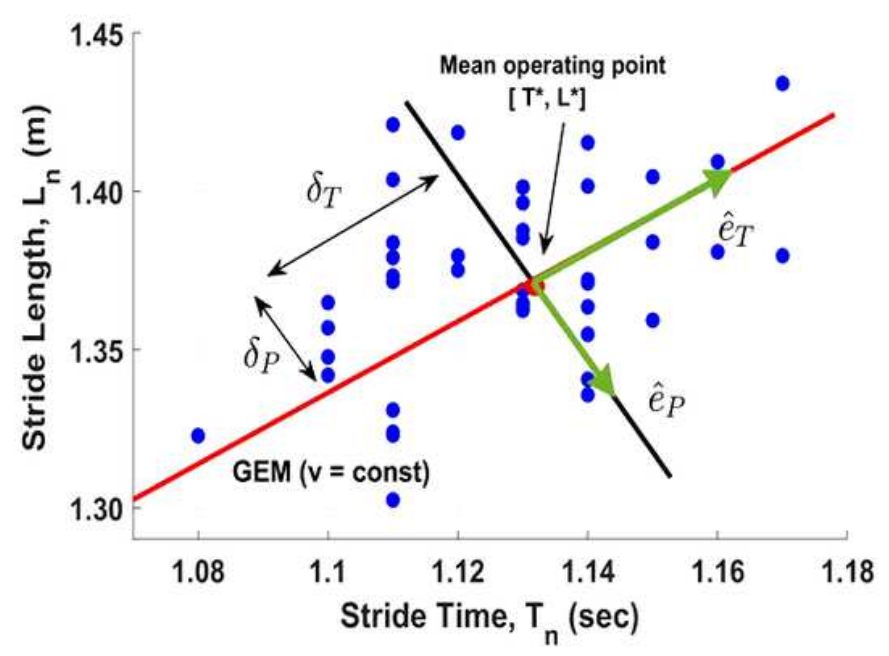

B
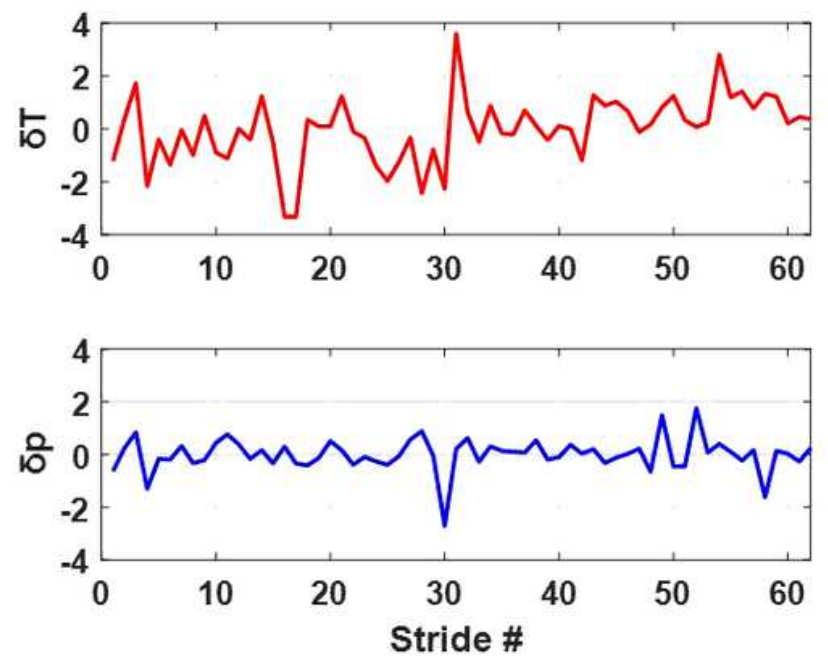

\section{Figure 1}

(A) Schematic depicting the goal equivalent manifold (GEM) for maintaining constant walking speed (v) using data from TF01. The red dot is the preferred mean operating point. Each blue dot represents the combination of stride time and stride length for an amputee participant with walking speed at $1.2 \mathrm{~m} / \mathrm{s}$. Dots that lie exactly on the red diagonal line (GEM) achieved the same speed and satisfied the goal. (B) The time series of $\delta T$ and $\delta p$ deviations for the data set shown in Figure $1 \mathrm{~A}$. 


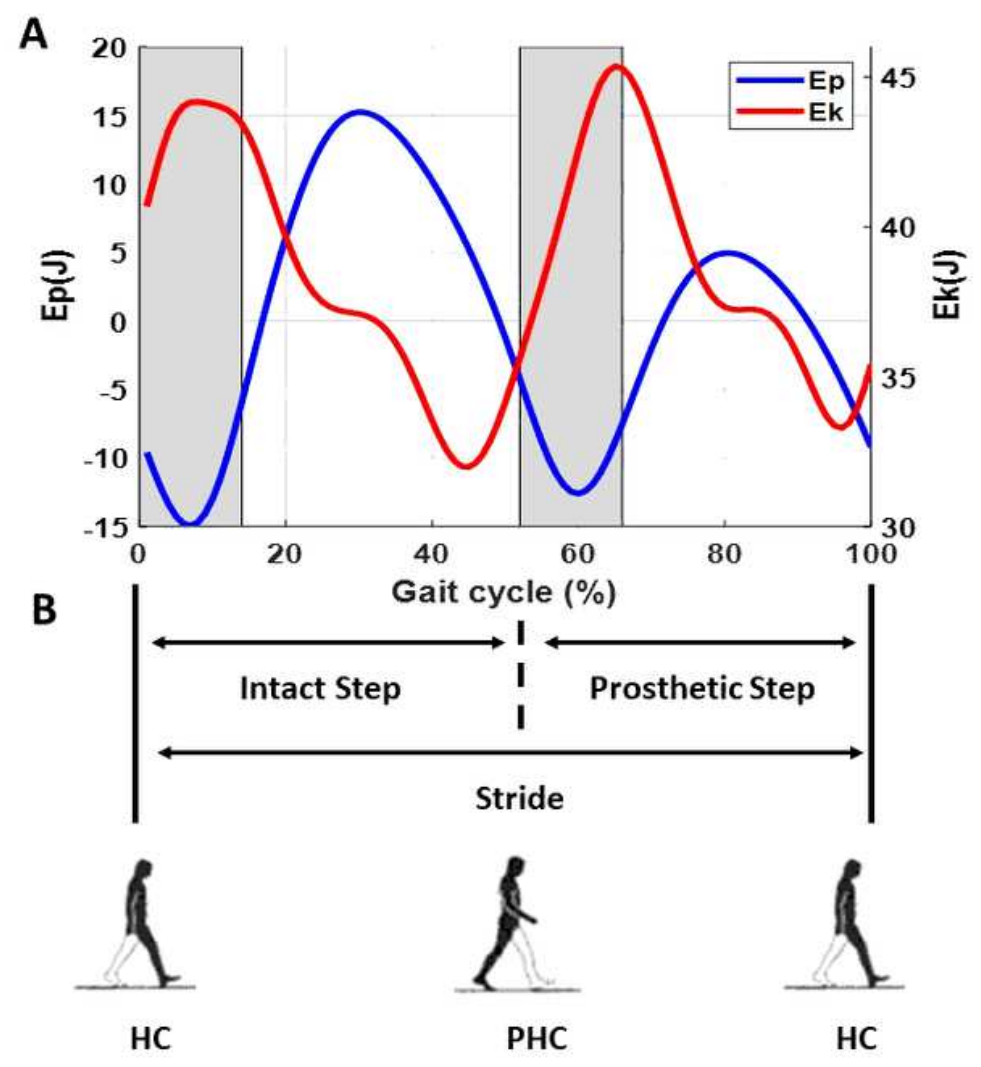

Figure 2

.(A) Example of Ep and Ek oscillations are plotted as a function of gait cycle duration. The shaded area indicates the periods of double support. The plot shows an out-of-phase oscillation of kinetic and potential energy, allowing energy exchange to occur between Ek and Ep . (B) Demonstration the stride and step (prothesis/intact step) period determined in this study. $\mathrm{HC}$ is heel contact of intact limb; PHC is heel contact of prosthetic limb. Stride period is determined from $\mathrm{HC}$ to the next HC. Prosthetic step is determined from $\mathrm{PHC}$ to $\mathrm{HC}$ where prosthetic limb leads, intact limb trails, and single support is on the prosthetic limb. Intact step is determined from HC to the PHC where intact limb leads, prosthetic limb trails, and single support is on the intact limb. 

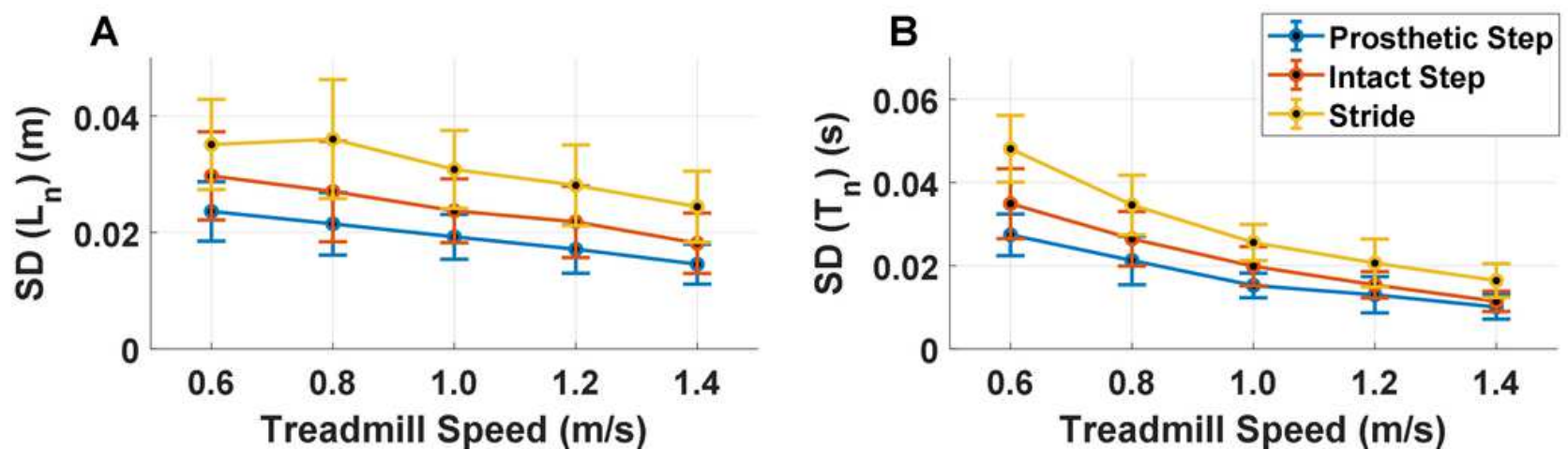

Figure 3

Primary gait parameters. Standard deviations (A, B) for stride and step length ( $\mathrm{Ln}$ ) and time (Tn) as a function of walking speed from $0.6 \mathrm{~m} / \mathrm{s}$ to $1.4 \mathrm{~m} / \mathrm{s}$. Error bars indicate between subject $95 \%$ confidence intervals at each speed. The SD of length and the SD of time in stride and step length linearly decreased along with speed.

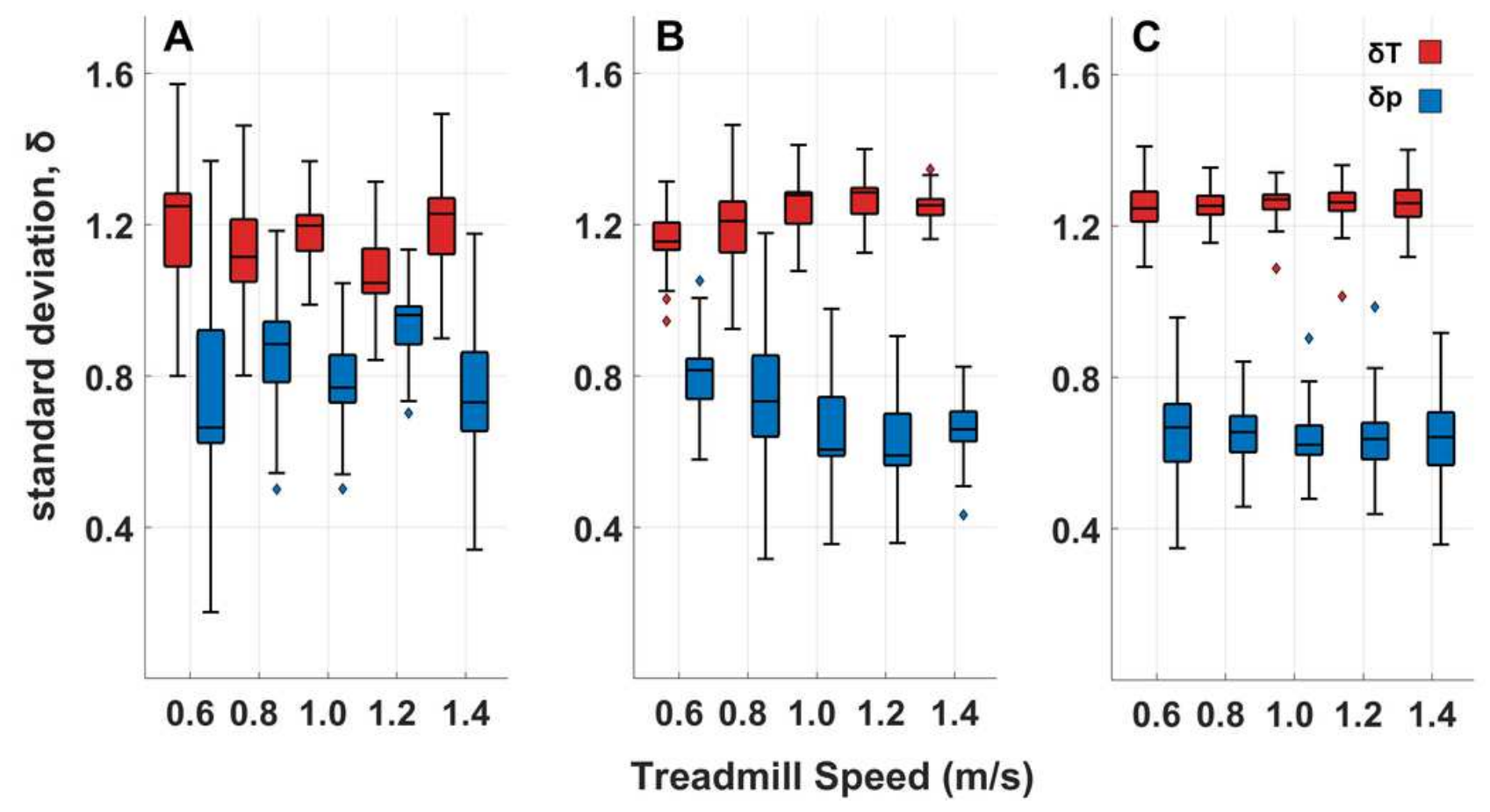

Figure 4 
GEM-Based Decomposition of Gait Variability. (A). Standard deviations for all $\delta T$ and $\delta p$ time series at all 5 walking speeds calculated from each stride period. (B). Standard deviations for all $\delta T$ and $\delta p$ time series at all 5 walking speeds calculated from each prosthetic step. (C). Standard deviations for all $\delta T$ and $\delta p$ time series at all 5 walking speeds calculated from each intact step.

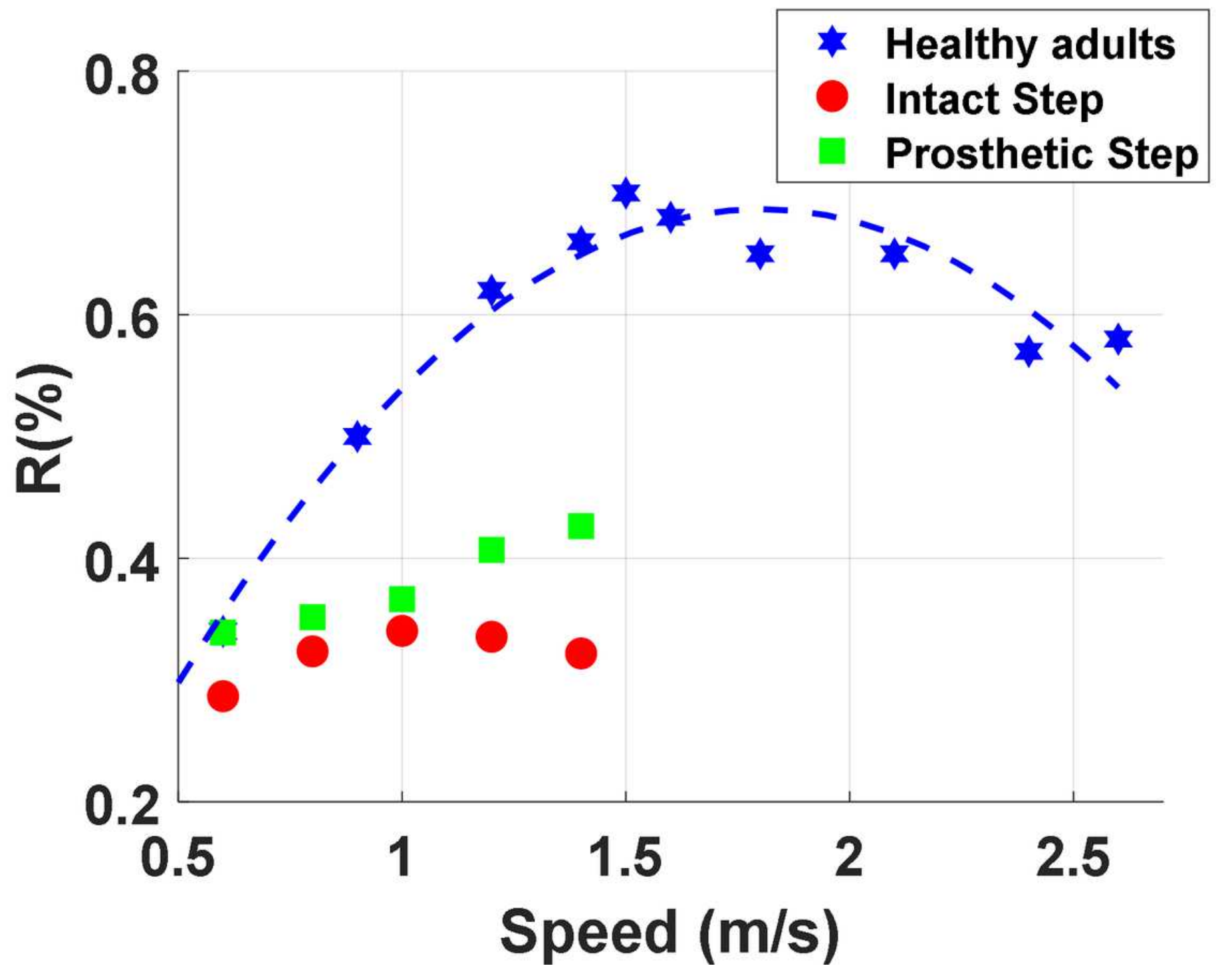

Figure 5

Energy recovery rate $(\mathrm{R})$ are plotted as a function of walking speed for healthy adults (hexagram), Intact Step (circles) and Prosthetic Step (square). Adult data were reproduced from Willems et al., 1995. 